Article

\title{
Heavy Quark Symmetry and Fine Structure of the Spectrum of Hadronic Dark Matter
}

\author{
Vladimir Kuksa *(D) and Vitaly Beylin \\ Research Institute of Physics, Southern Federal University, Pr. Stachky 194, 344090 Rostov-on-Don, Russia; \\ beylinv@sfedu.ru \\ * Correspondence: vkuksa47@mail.ru
}

Received: 13 October 2020; Accepted: 18 November 2020; Published: 20 November 2020

\begin{abstract}
We analyze the structure of excited states of new heavy hadrons in the scenario with hadronic dark matter. Fine mass-splitting in a doublet of new mesons stipulates the existence of charged metastable heavy mesons. We describe the structure of new meson excited states in the framework of the heavy quark effective theory. Phenomenological consequences of fine and hyperfine splitting are considered in the hadronic dark matter scenario and beyond.
\end{abstract}

Keywords: hadronic dark matter; hyperfine splitting; luminosity

\section{Introduction}

Strongly interacting massive particles (SIMP) as dark matter (DM) candidates are the subject of urgent discussion in literature during the last years [1-4]. The interest in SIMP as the DM candidate increased after the appearance of a strong restriction on the weakly interacting massive particles (WIMP) scenario [5]. Hadronic dark matter (HDM) is one of the simplest and most natural variants of the SIMP scenario. In this scheme, DM particles are heavy hadrons which consist of new heavy quarks, $Q$, and ordinary ones, $q$. The most developed extensions of the Standard Model (SM) with new quarks were considered in Refs. [6] (and references therein). Some peculiar properties of new heavy mesons and low-energy phenomenology of the HDM scenario were considered in Refs. [4,6,7]. It was shown in these works that new quarks have vector-like interaction with gauge bosons and the HDM scenario is not excluded by electro-weak (EW) restrictions on new physics and cosmochemical constraints on the relative concentrations of anomalous elements. In addition, the main properties of new hadrons, such as the value of mass, presence of metastable state for charged hadron and effect of fine splitting, were described in these references.

In this work, we consider the scenario with stable $S U(2)$-singlet quark (SQ) naturally following from the most investigated extensions of $\mathrm{SM}$, such as $E_{6}$ model or $S U(5)$ supersymmetric extension (for more details, see Refs. [4,6]). Note, these extensions have independent meaning as variants of realization of grand unification theory. Application of this scenario to the description of DM is not obligatory, however, it gives the simplest and most natural realization of the hadronic DM scenario. It is declared in Ref. [8] that the existence of cald DM in the framework of traditional gravity theory $(\Lambda \mathrm{CDM})$ is excluded with high significance level. Further, we consider the general scenario, where new heavy hadrons can form a subsystem of hidden sector, and a scenario with hadronic dark matter as the limiting case. Note, dark matter interpretation of new hadrons imposes the restriction on the value of hadron mass only, which can be used in the general analysis. The principal part of consideration does not depend on the status of new hadrons. The presence of a singlet quark in cosmic rays should be assumed without reference to DM, if we believe in the grand unification paradigm. The problem of the singlet quark was widely discussed in literature for several decades. The most attention was paid to the effect of mixing of this quark with ordinary ones to provide decay channels for the singlet quark. 
Here, we consider a scenario without mixing, then the singlet quark is absolutely stable due to baryon charge conservation. We should note, also, that the origin of singlet quark's mass, in contrast to masses of standard quarks, is not described by Higgs mechanism. In the early Universe, at the temperature above the mass $M$ of new quark $Q$, these quarks were in thermodynamic equilibrium with quark-gluon plasma due to annihilation $Q \bar{Q} \rightarrow g g, q \bar{q}$ and inverse processes. At the freezing out temperature, the rate of expansion exceeds the rate of annihilation of $Q \bar{Q}$-pairs, so they are frozen out. The mass of new quarks we have defined from the data on the DM relic density using the following equality:

$$
\left(\sigma(M) v_{r}\right)^{M o d}=\left(\sigma v_{r}\right)^{E x p}
$$

In Equation (1), the left part of the equality is the model value of annihilation cross-section and the right part follows from the data on the modern DM relic abundance of DM, $\left(\sigma v_{r}\right)^{\text {Exp }}=2 \times 10^{-9} \mathrm{GeV}^{-2}$. The total cross-section of the strong channels (new quarks posses standard QCD interaction) of annihilation $Q \bar{Q} \rightarrow g g, q \bar{q}$ is calculated in [6]:

$$
(\sigma(M))^{M o d}=\sigma(Q \bar{Q} \rightarrow g g, q \bar{q}) \approx \frac{44 \pi}{9} \frac{\alpha_{s}^{2}}{M^{2}} .
$$

Using the expression (2) and equality (1), we estimate the new quarks mass as $M \approx 10 \mathrm{TeV}$. It defines the mass scale of new hadrons. From this estimation, it follows that the freezing out temperature $T_{f} \approx M / 30 \approx 300 \mathrm{GeV}$, which is much larger than the QCD phase transition temperature, $T_{Q C D} \approx 150 \mathrm{MeV}$. Therefore, the stage of hadronization of usual and new heavy hadrons begins much later than the freezing out one. Note, if DM status of new quarks is not assumed, then the above estimation gives a lower bound on mass, $M>10 \mathrm{TeV}$. After phase transition, new heavy quark $Q$ combines with ordinary light quark $q$ into a new heavy $Q q$-hadrons. In the Universe with baryon asymmetry, it is possible the formation of meson states $(q \bar{Q})$ and baryon states $(q q Q)$ with unit electrical charge. Here, we consider the meson states only, while more complicated states were considered in Ref. [6]. In Ref. [7], the scenario with asymmetry in the sector of new quarks (only antiquarks $\bar{Q}$ exist) was considered, where new heavy baryons were absent. In this work, we analyze effects of fine and hyperfine mass-splitting in detail and consider principal consequences of these effects.

The paper is organized as follows. In the Section 2, we consider the effect of fine and hyperfine splitting in the spectrum of masses of standard and new heavy-light mesons. In Section 3, we take into account some constraints on the scenario following from the cosmological data, and analyze the main phenomenological consequences of fine and hyperfine splitting. Discussion and some conclusions are presented in Section 4.

\section{Fine and Hyperfine Structure of the Mass Spectrum of New Heavy Mesons}

\subsection{Fine Isotopic Structure of the Doublet $\left(M^{0}, M^{-}\right)$}

New heavy mesons are composite two-quark states $M=(q \bar{Q})$, where $q$ is the light standard quark and $Q$ is the new (super) heavy quark. Therefore, these heavy-light mesons are near asymptotical objects of heavy quark effective theory (HQET) [9-13]. This theory successfully describes standard heavy-light (HL) mesons, such as $K, D$ and $B$ mesons. The base idea of HQET is the heavy quark symmetry which takes place at $m_{Q_{1}}, m_{Q_{2}} \gg \Lambda_{Q C D}$ [10]. In this case, the heavy degrees of freedom split off and can be integrated out; that is, in the limit $m_{Q} \rightarrow \infty$, the solutions of the QCD equations become independent on the mass and spin of the heavy quark. This effect makes it possible to establish the relation between some characteristics of heavy-light mesons. In this work, with the help of the HQET approach, we establish the relations between mass-splitting in a set of standard HL mesons ( $\mathrm{D}$ and $\mathrm{B}$ mesons) and new heavy mesons. These relations lead to the effects of fine and hyperfine splitting, which can manifest itself as the existence of a metastable charged meson and luminosity of hadronic DM. 
Firstly, we consider the mass-splitting in the doublets of new mesons, $M=\left(M^{0}, M^{-}\right)$, where neutral and charged components contain different light quarks. In the case of up quark $Q=U$, quark content of neutral and charged mesons are as follows: $M^{0}=(u \bar{U})$ and $M^{-}=(d \bar{U})$. In the case of down quark $Q=D$, the contents are: $M^{0}=(d \bar{D})$ and $M^{+}=(u \bar{D})$. The value of mass-splitting, $\Delta m=m\left(M^{-}\right)-m\left(M^{0}\right)$ is defined by differences of binding energy of these states and the masses of light quarks, $u$ and $d$. Binding energy of new heavy mesons is caused both by strong and electro-weak interactions, while the definition of masses of light quarks is model dependent (for example, the current-quark masses, $m_{u}=2.3 \mathrm{MeV}$ and $m_{d}=4.8 \mathrm{MeV}$ [14], are much less constituent-quark mass, $M_{u} \approx M_{d} \sim 10^{2} \mathrm{MeV}$ ). It is difficult to account for all of these factors; therefore, we use the experimental data on the mass-splitting in standard heavy-light mesons, $K, D$ and $B$. Then, with the help of the heavy quark symmetry (see, for example, Ref. [10]) we extrapolate the values of resulting effect to the doublet of new heavy mesons. From the experimental data on the masses of neutral and charged components of standard doublets [14], it follows:

$$
\Delta m_{K} \approx-3.9 \mathrm{MeV} ; \Delta m_{D} \approx+4.8 \mathrm{MeV} ; \Delta m_{B} \approx-0.3 \mathrm{MeV} .
$$

Using these data, one can makes the following conclusions: there is no monotonous behavior of the value $\Delta m$ with respect to the meson mass; the value of mass-splitting is positive, $\Delta m>0$, for the case of $D$-meson (up-type of heavy quark, $c$-quark) and negative, $\Delta m<0$, for the cases of $K$ and $B$ mesons (down-type of heavy quarks, $s$ - and $b$-quark); absolute value of mass-splitting is on the order of $\mathrm{MeV}$. The existence of heavy-quark symmetry makes it possible to apply these conclusions to the case of new heavy doublet $\left(M^{0}, M^{-}\right)$. The term "fine mass-splitting", in this case, designates the inequality $\Delta m \ll m(M)$, where $\Delta m \sim 1 \mathrm{MeV}$ and $m(M) \sim 10^{7} \mathrm{MeV}$. From these preliminary assumptions, one important conclusion for the hadronic DM scenario follows: new quark, which forms the lightest neutral stable state, should be up-type quark, $U$ (see, also, Ref. [6]). Moreover, due to the absence of strong channels of new mesons decay, we can assume the unique weak decay channel of charged component:

$$
M^{-} \rightarrow M^{0} e^{-} \bar{v}_{e}
$$

This decay process was described in Ref. [6], where the width and lifetime was calculated with help of effective low-energy Lagrangian of M-particles and $\mathrm{W}$-boson weak interactions. The structure of the process can be represented by one diagram with $W$-boson in the intermediate state, $M^{-} \rightarrow M^{0} \mathrm{~W}^{-} \rightarrow M^{0} e^{-} \bar{v}_{e}$. The first step of decay $M^{-} \rightarrow M^{0} \mathrm{~W}^{-}$is described by effective differential vertex $M^{-} M^{0} W^{-}$with effective coupling $G=g /(2 \sqrt{2})$ [6], where $g$ is standard weak coupling. The second step is described by standard vertex $W^{-} e \bar{v}$ with vector and axial parts. Straightforward calculation of width of the decay $1 \rightarrow 3$ with the help of the standard method leads to the following expression:

$$
\Gamma\left(M^{-}\right) \approx \frac{G_{F}^{2}}{60 \pi^{3}}\left[(\Delta m)^{5}-m_{e}^{5}\right] .
$$

From Equation (5), one can see that the reaction has a threshold, and the condition of destabilization (there are no stable new charged mesons) is described by inequality $\Delta m>m_{e}$. From the third assumption, $\Delta m$ is on the order of $\mathrm{MeV}$, it follows that the value of width can be very small and $M^{-}$can be long-lived. In particular, at $\Delta m=1 \mathrm{MeV}$, the lifetime $\tau=1 / \Gamma \sim 10^{5} \mathrm{~s}$. Moreover, in the limit $\Delta m \rightarrow m_{e}$, the charged particle $M^{-}$has an arbitrary large lifetime. The presence of a long-lived charged partner of DM particle is one of the most specific features of the hadronic DM scenario. Note, the possibility of existence of charged metastable heavy hadros was analyzed in [7], where its indirect experimental evidences and signals in cosmic ray physics were considered.

The presence of metastable charged particles in the early Universe can be impacted by their charged decay products on the parameters of cosmic microwave background (CMB) and "Big Bang" nucleosynthesis (BBN). Possible constraints on the HDM scenario will be discussed in the next 
section, where we present the phenomenological consequences of the fine structure effect and possible manifestation of metastable charged hadrons.

\subsection{Hyperfine Structure of Excited States of New Heavy Mesons}

Effect of hyperfine splitting principally differs from the fine mass-splitting, but it can be directly described in the framework of heavy quark effective theory (HQET) $[10,13]$. The effect of fine splitting is caused by the change of light quark content $\left(q \rightarrow q^{\prime}\right)$, and heavy quark symmetry allows us to extrapolate the main characteristics of this effect on new heavy mesons. Hyperfine splitting relates to the mass-splitting of excited states of the mesons with the same quark content. As was shown in the framework of HQET, this effect disappears in the limit of infinite mass of meson, when heavy quark symmetry becomes exact. Here, we consider the hyperfine splitting effect for the case of the lowest excited and ground states.

First of all, we briefly represent the base statements of HQET, which usually are applied to the description of standard heavy-light (HL) mesons $K, D$ and $B$ (see the Refs. [10-13] and references therein). Heavy quark symmetry is the base idea of HQET [10], which becomes exact in the limit of infinite mass of meson, $m(M) \approx m_{Q} \rightarrow \infty$. For the realistic case of finite masses of standard mesons, this symmetry is approximate and in the framework of HQET is realized as systematic approximation to full QCD. An account of corrections is fulfilled with the help of small parameter $\Lambda / m_{Q}$, where $\Lambda$ is the scale of the light degree of freedom. It was shown in Refs. [12,13], that by the procedure of integrating off the heavy quark degree of freedom, the expression for potential (Lagrangian or Greens function) can be represented in terms of expansion on small parameter $\Lambda / m_{Q}$. For example, full quasi-potential is as follows [13]:

$$
U(q, Q)=U_{0}(q)+\frac{1}{m_{Q}} U_{1}(q, Q)+\frac{1}{m_{Q}^{2}} U_{2}(q, Q)+\ldots,
$$

where $U_{0}(q)$ does not depend on some characteristics of heavy quark $Q$, while the rest of the terms of expansion do. This effect has some principal aspects which are important in the description of super-heavy mesons. The first part in Equation (6), $U_{0}(q)$, does not depend on mass and spin of the heavy quark; that is, it describes the set of degenerate states. Independence on mass $m_{Q}$ leads to the existence of, so called, heavy quark symmetry, which becomes exact at $\Lambda / m_{Q} \rightarrow 0$ and allows us to extrapolate some properties of heavy-light (HL) standard mesons to the case of super-heavy new mesons. Independence on spin orientation of heavy quark in $U_{0}(q)$ leads to spin-spin and spin-orbital degenerations, which are removed by an account of the rest terms in Equation (6). Another aspect refers to the small parameter $\Lambda / m_{Q}$ for the case of heavy quarks which leads to the hyperfine splitting of degenerated states.

Further, we describe the effect of spin-spin and spin-orbital splitting in the HL mesons and estimate its value for new heavy mesons with the help of HQET. The ground and the lowest excited states of the meson system $M(q \bar{Q})$ have the following quantum numbers: $J^{P}=0^{-}$and $J^{P}=1^{-}$. Here, $J$ is the full angle moment, $P=(-!)^{L+1}$ is parity and $L$ is orbital moment, which is zero, $L=0$, for the case under consideration. Then, $J=0$ when spins of quarks $q$ and $Q$ are antiparellel; that is $\vec{S}_{Q}=-\vec{S}_{q}$, and $J+1$ for the case of parallel spins, $\vec{S}_{Q}=\vec{S}_{q}$. As was noted earlier, the dominant term of potential (Lagrangian) does not contain the spin of the heavy quark, $\vec{S}_{Q}$, so, in this approximation, the states $0^{-}$(pseudoscalar) and $1^{-}$(vector) are degenerated with respect to the energy level (mass). It was explicitly shown in Ref. [13], that $L_{1}(q, Q)$ depends on the spin of heavy quark, $\vec{S}_{Q}$, in the form of scalar products $\left(\vec{S}_{Q} \cdot \vec{S}_{q}\right)$ and $\left(\vec{S}_{Q} \cdot \vec{L}\right)$, which describe spin-spin and spin-orbital interactions. In the case $L=0$, the splitting is caused by the spin-spin interaction only. The effect of splitting and the low-energy spectrum for the case of standard HL mesons usually are described in the quasi-potential approach, where complicated equations are solved numerically [13]. Theoretical results are in good agreements with experimental data on standard HL mesons; that is, heavy quark symmetry and HQET are relevant for standard mesons, which are not too heavy. Moreover, the relations between the values 
of splitting are in accordance with heavy quark symmetry, so, this symmetry is not strongly violated. Such effect can be illustrated by the following phenomenological relation [12]:

$$
\Delta m^{2}=m^{2}\left(1^{-}\right)-m^{2}\left(0^{-}\right) \approx 2 m_{Q}\left[m\left(1^{-}\right)-m\left(0^{-}\right)\right] \approx \text { Const, }
$$

where we used approximate equality for HL mesons, $m\left(1^{-}\right)+m\left(0^{-}\right) \approx 2 m_{Q}$. From the experimental data on the masses of the mesons $M=(B ; D ; K)$ [14], it follows the validity of Equation (7) with good accuracy, $\Delta m^{2}=(0.53 ; 0.54 ; 0.55) \mathrm{GeV}^{2}$. As it follows from the HQET (see Equation (6)), the accuracy of Equation (7) increases with increasing of mass $m_{Q}$. Therefore, we can apply this relation in order to estimate the splitting of super-heavy mesons in ground, $M$, and excited, $M^{*}$, states. Using Equation (7), the relation between mass-splitting for $B$ and $M$ mesons can be represented in the form:

$$
\frac{\delta m_{M}}{\delta m_{B}}=\frac{m\left(M^{*}\right)-m(M)}{m\left(B^{*}\right)-m(B)} \approx \frac{m_{b}}{m_{U}}
$$

where $M$ and $M^{*}$ designate the mesons with quantum numbers $0^{-}$and $1^{-}$. Using the values $m_{b} \approx 4 \mathrm{GeV}, \delta m_{B} \approx 45 \mathrm{MeV}$ (see Ref. [14]) and our estimation $m_{Q}=10^{4} \mathrm{GeV}$, from Equation (8) we get the value of mass-splitting, $\delta m_{M} \approx 2 \mathrm{keV}$. Thus, from the experimental data on mass-splitting of B-mesons and principal statements of HQET, it follows the effect of hyperfine splitting in the system of M-mesons. Here, we should note that similar mass-splitting is discussed in Refs. [15,16], where this splitting was introduced in phenomenological way to explain XENON1T excess [17].

Now, we briefly consider the orbitally excited states, $M_{1}$ and $M_{1}^{*}$, with orbital moment $L=1$ and parity $P=(-1)^{2}=+1$. The values of summary angular moment $\vec{j}=\vec{L}+\vec{s}_{q}$ and total angular moment $\vec{J}=\vec{j}+\vec{s}_{Q}$ are the following:

$$
\begin{aligned}
& \text { 1) } j=1-\frac{1}{2}=\frac{1}{2} ; J=j-\frac{1}{2}=0 M\left(0^{+}\right) ; J=j+\frac{1}{2}=1 M\left(1^{+}\right) ; \\
& \text {2) } j=1+\frac{1}{2}=\frac{3}{2} ; \quad J=j-\frac{1}{2}=1 M^{\prime}\left(1^{+}\right) ; J=j+\frac{1}{2}=2 M\left(2^{+}\right)
\end{aligned}
$$

The states $M\left(0^{+}\right), M\left(1^{+}\right)$and $M^{\prime}\left(1^{+}\right), M\left(2^{+}\right)$are mutually degenerate in zero approximation due to heavy quark symmetry. Really, both components in these pairs differ by the orientation of heavy quark spin, $\vec{S}_{Q}$ and the splitting of the levels is caused by the second term of expansion (see Equation (6)). We should note that analogs of $M^{\prime}\left(1^{+}\right)$and $M\left(2^{+}\right)$are the mesons $B_{1}^{0}$ and $B_{2}^{* 0}$ with the masses $m_{1}=5723.5 \mathrm{MeV}$ and $m_{2}=5743 \mathrm{MeV}$ [14]. Using this data and theoretical estimations of the masses of B-analogs of $M\left(0^{+}\right)$and $M\left(1^{+}\right)$[13], we can conclude that there are two doublets of degenerate states of M-mesons with positive parity, which have the masses $m\left(M^{+}\right) \approx m_{Q}+0.5 \mathrm{GeV}$ and hyperfine splitting on the order of $1 \mathrm{keV}$. The interval between these doublets for the case of B-mesons, which is defined by the first term in Equation (6), was numerically calculated in Ref. [13], $\Delta m \approx 20 \mathrm{MeV}$. In accordance with heavy quark symmetry, the same value of the interval should be found for the case of M-mesons spectrum. The above described features of M-mesons spectrum follow from the heavy quark symmetry and HQET estimations.

\section{Phenomenological Consequences of Fine and Hyperfine Structure}

Existence of excited states of new heavy hadrons in the hadronic DM scenario has some important phenomenological consequences. Namely, recombination of excited states can lead to the luminosity of HDM during its interaction with ordinary matter (see, for example, Ref. [18]). At last, underground processes of excitation and recombination of hadronic DM particles, which are absorbed by our planet, in principle, can generate some signals in detectors, for example, such as registered by XENON1T [17]. Here, we analyze these effects and briefly describe corresponding processes, their signals and possible constraints on the HDM scenario. 
As was shown above, fine splitting in the new mesons doublet, $\left(M^{0}, M^{-}\right)$, leads to the existence of metastable charged heavy meson $M^{-}$. The presence of these particles in the early Universe can affect on the parameters of CMB and BBN $[19,20]$. Here, we consider the possible constraints on the HDM scenario which follow from cosmological data on CMB and BBN parameters. Constrains on long-lived new particles, which were derived in $[19,20]$, mainly stipulated by the electromagnetic (e.m.) product of annihilation or decay, for example, $\chi \rightarrow e^{+} e^{-} ; \gamma$, where $\chi$ is unstable component of DM. The effect depends on the value of injected electromagnetic energy to the plasma and on the fraction of unstable component of DM $[19,20]$. The scenario under consideration differs from this picture due to some principal peculiarities of hadronic DM. At the beginning of the hadronization, fractions of neutral, $M^{0}=(u \bar{U})$, and charged, $M^{-}=(d \bar{U})$, components of new mesons are defined by relative fraction of light quarks, $u$ and $d$; that is, the fraction can be an order of unit. Later on, this fraction exponentially decreases $n^{-} / n^{0} \sim \exp (-t / \tau)$, however, the rate of decreasing is partially compensated by its production in the process $e^{-} M^{0} \rightarrow v_{e} M^{-}$. The rate of the last process, in turn, depends on the value of threshold $\Delta m$ and temperature of plasma. Effective energy density parameter $\Theta$, which describes the fraction of e.m. energy injected into plasma, is the relative amount of energy released as the e.m. energy in one decay, normalized to the current total cold DM abundance (see Ref. [19]). For the decay $M^{-} \rightarrow e^{-} M^{0}$, it can be approximately defined as ratio of mass splitting (injected energy) and mass of DM particles, $\Theta \sim \Delta m / M \sim 10^{-7}$. From the constrains on parameter $\Theta$ in Refs. [19,20], it follows the approximate constraint on lifetime of charged particle $M^{-}, \tau_{M}<10^{12} \mathrm{~s}$. This upper limit corresponds to the value of mass-splitting $\Delta m \approx m_{e}$. This constraint, however, does not account for the impact of charged component $M^{-}$on $\mathrm{CMB}$ at the beginning stage of hadronization at $t<\tau_{M}$. At the case of large mass-splitting, $\Delta m \sim 10 \mathrm{MeV}$, the lifetime $\tau_{M} \sim 1 \mathrm{~s}$ and this impact is small. To account for this impact in the case of mass-splitting $\Delta m \sim 1 \mathrm{MeV}$, we need to modify the above described scheme.

In the presence of excited states of new hadronic particles, transitions between these states are accompanied by absorption and radiation of photons in the $\mathrm{keV}$ spectral range. It corresponds to $\gamma$-rays with wavelength $\lambda \sim 10^{-9} \mathrm{~cm}$. Assuming that characteristic size of M-mesons, $R_{M}$, is not greater than nucleon radius, that is $R_{M} \leq 10^{-13} \mathrm{~cm}$, we get strong inequality $\lambda_{t r} \gg R_{M}$. Therefore, interaction of such photons with neutral composite system $M^{0}$ is defined by higher terms of multipole expansion of charge distribution inside meson and is negligible. There is, however, another mechanism of M-mesons exciting through low-energy interaction with nucleons or leptons at small momentum transfer. Such processes can be caused by neutral transitions through the exchange of neutral light mesons, $\omega, \rho^{0}$, or standard bosons $\gamma, \mathrm{Z}$ in $t$-channel reactions:

$$
M^{0} N \longrightarrow M^{0 *} N\left(\omega, \rho^{0} \text { - exchange }\right) ; M^{0} l \longrightarrow M^{0 *} l,(\gamma, Z \text { - exchange }) .
$$

Kinematics of these quasi-elastic scatterings are the same as for the elastic scattering, but the vertices are different. The vertex of $M M V$-interaction has a differential form, while the vertex of $M M^{*} V$ is not, because the field $M^{*}=M\left(1^{-}\right)$has vector character. Corresponding Lagrangians are as follows:

$$
L(M M V)=i g_{s} V^{\mu}\left(M \partial_{\mu} \bar{M}-\partial_{\mu} M \bar{M}\right) ; L\left(M M^{*} V\right)=i g_{v} V^{\mu} M_{\mu}^{*} M,
$$

where $M_{\mu}^{*}=M_{\mu}\left(1^{-}\right)$is vector state and $g_{s}, g_{v}$ are effective couplings, which in the general case depend on momentum transfer as form-factor. Note, the form of the Lagrangian $L\left(M M^{*} V\right)$ in (11) is the simplest one, however, it is not unique (see Equation (15) below). The cross-section of elastic scattering of the type $N M \rightarrow N M$ was presented in Refs. [4,21]. The expression for cross-section of strong low-energy scattering is as follows:

$$
\sigma(M N \rightarrow M N)=\frac{g^{4} m_{N}^{2}}{16 \pi m_{\rho}^{4}}\left(1+\frac{k_{N}}{3}\right)^{2},
$$


where $M=\left(M^{0}, M^{-}\right), N=(p, n), g^{2} / 4 \pi \approx 3.4$ and $k_{N}= \pm 1$ for the case of $p$ or $n$. In particular, proton scattering on DM particle $M^{0}$ has a rather large value of cross-section, $\sigma\left(p M^{0} \rightarrow p M^{0}\right) \approx$ 0.9 barn. Formally, this cross-section does not account for transition $M^{0} \rightarrow M^{0 *}$ directly, because the last reaction is accompanied by the change of mutual orientation of spins of light, $\vec{s}_{q}$, and heavy, $\vec{s}_{Q}$, quark. Taking into account hyperfine mass-splitting, that is the equality of final phase spaces, we should expect that the relation of corresponding transition probabilities to be near the same, if $g_{s} \approx g_{v}$. From the structure of one-meson exchange reaction, it follows that intermediate mesons interact mainly with light quark in M-particle, while heavy quark $\bar{U}$ has spectator status. As a result of this interaction, light quark can convert orientation of its spin and $M\left(0^{-}\right)$transits to $M\left(1^{-}\right)$. Therefore, this transition is not suppressed and can lead to consequent process of recombination, $M\left(1^{-}\right) \rightarrow M\left(0^{-}\right) \gamma$, with radiation of photons having energies near $2 \mathrm{keV}$.

Now, we consider the constraints on hadronic DM which strongly interact with ordinary baryons. The presence of such DM particles directly impact on the parameters of BBN and $\gamma$-spectrum of cosmic rays (CR) in the Galaxy [22]. In this work, the constraints were derived on the relation $R=\sigma\left(\mathrm{cm}^{2}\right) / M_{D M}(\mathrm{~g})$, where $\sigma$ is cross-section of DM-baryon scattering (in $\mathrm{cm}^{2}$ ) and $M_{D M}$ is the mass of DM particle (in g). The constraints are as follows [22]:

$$
B B N: R<10^{8} \mathrm{~cm}^{2} \mathrm{~g}^{-1} ; \quad C R: R<5 \times 10^{-3} \mathrm{~cm}^{2} \mathrm{~g}^{-1} .
$$

Therefore, the second restriction is much more stringent and we compare it with model result. In our consideration, the value of mass is $M_{D M} \approx 10^{4} \mathrm{GeV} \approx 10^{-20} / 0.56 \mathrm{~g}$, and cross-section $\sigma \sim$ $10^{-24} \mathrm{~cm}^{2}$. Thus, the model relation $R \approx 5.6 \times 10^{-5} \mathrm{~cm}^{2} \mathrm{~g}^{-1}$ does not contradict to the $\mathrm{CR}$ restriction. There are more stringent constraints on the HDM scenario which follow from the observations of interaction between DM and baryon from X-ray Quantum Calorimetry Experiments (XQC) [23]. It excludes DM with mass $M=10^{4} \mathrm{GeV}$ in the range of cross-section $\left(10^{-27}-10^{-21}\right) \mathrm{cm}^{2}$, so, the model estimation $\sigma \sim 10^{-24} \mathrm{~cm}^{2}$ falls into this range. However, we note that the model value of cross-section is estimated in meson-exchange approach, which is valid at small momentum transfer. In Ref. [6], we have shown that for the case of DM-nucleon non-relativistic collisions, the momentum transfer is $Q^{2} \approx$ $2 m_{N}^{2} v^{2}(1-\cos \theta)$, where $m_{N}$ is nucleon mass and $v \sim 10^{-3}$. Therefore, for pure DM-nucleon collisions $Q^{2} \sim 1 \mathrm{MeV}^{2}$, this approach is valid. In the case of XQC measurements, $Q^{2} \approx 2 m_{T}^{2} v^{2}(1-\cos \theta)$, where $m_{T}$ is the mass of target nuclear, $m_{T} \sim 10^{2} m_{N}$ (detector material). Thus, $Q^{2} \sim 10^{4} \mathrm{MeV}^{2}$ and the model estimation are invalid at this momentum transfer. We should modify the model of DM-nucleon interaction for the case of large momentum transfer (see, also, the comments in the introduction concerning DM status of new hadrons). The more proper measurements and constraints are considered in Ref. [24] for the case of cosmic ray interaction with DM. The constrains were developed using NFW and Moore DM density profiles and new data from the Fermi gamma ray space telescope. Here, we use the upper constraint with Moore profile (which is more stringent): $\sigma_{N x}=9.3 \times 10^{-30} m_{x} \mathrm{~cm}^{2} \mathrm{GeV}^{-1}$. At $m_{x}=10^{4} \mathrm{GeV}$, we get $\sigma<10^{-25} \mathrm{~cm}^{2}$, which excludes the model again. Here, we note that we describe the DM-nucleon interaction in the meson-exchange approach using the coupling constant, which was determined in low-energy hadrons interaction $\left(g^{2} / 4 \pi=3.4\right)$. Thus, the model assumption concerning the value of coupling is not justified, and from the experiment we get the constraint on this parameter: $g^{2} / 4 \pi<1$. Here, we should note that, in spite of large value of DM-nucleon cross-section, it is difficult to detect directly DM particles by underground detectors (see the end of this Section). It is more perspective to register the effect of dark kinetic heating of a neutron star (so called, neutron star capture). As was shown in Ref. [25], this method is more sensitive than direct detection one, particularly, in the light and super-heavy mass region.

Further, we consider the processes of electron scattering on DM particles $e M^{0} \rightarrow e M^{0} ; e M^{0 *}$, where exchange of $\gamma$ or $Z$-boson in $t$-channel takes place. At low energy, the contribution of the channel with $Z$-boson exchange is suppressed by the factor $q^{2} / M_{W}^{2}$, where $q$ is momentum transfer, and the channel with $\gamma$-exchange is dominant. Further, we consider this process in analogy with 
process of electron scattering on ordinary mesons. Invariant transition matrix element usually is represented in the form:

$$
M_{f i}=K \frac{e^{2}}{q^{2}}\left(\bar{U}_{e}^{f} \gamma^{\mu} U_{e}^{i}\right)\left(\bar{U}_{M}^{f} \Gamma_{\mu} U_{M}^{i}\right)
$$

where $K$ is the normalizing coefficient, $U_{e}$ and $U_{M}$ are field functions of electron and $M$-meson. The first term in brackets is the lepton current and the second one is the hadron current. The structure of vertex operator in hadron current, $\Gamma_{\mu}$, is defined by the type of mesons $M$ in initial and final states. For the case of elastic scattering of electron on spin-zero particle $e M\left(0^{-}\right) \rightarrow e M\left(0^{-}\right)$, the expression for vertex structure is well-known, $\Gamma_{\mu}=\left(p_{1}+p_{2}\right)_{\mu} f_{+}\left(q^{2}\right)$. Therefore, the process is defined by one function of momentum transfer $q^{2}$, which is usually named as hadron form-factor. In the case of hadron with spin $s=1 / 2$ (fermion), the vertex operator contains two independent real (at $\left.q^{2}<0\right)$ form-factors. The transition of type $M\left(0^{-}\right) \rightarrow M\left(1^{-}\right)$is described in the general case by three form-factors (see, for example, [26]):

$$
\begin{aligned}
\left\langle M(K, e)\left|\Gamma_{\mu}\right| M(P)\right\rangle & =i g\left(q^{2}\right) \epsilon_{\mu \nu \rho \sigma} e^{\nu}(P+K)^{\rho}(P-K)^{\sigma} \\
& +f\left(q^{2}\right) e_{\mu}+a_{+}\left(q^{2}\right)(e \cdot P)(P+K)_{\mu} .
\end{aligned}
$$

Thus, to calculate the probability of electromagnetic transitions of type $M\left(0^{-}\right) \rightarrow M\left(0^{-}\right)$and $M\left(0^{-}\right) \rightarrow M\left(1^{-}\right)$, we should define the form-factors of new heavy mesons. The excited state $M\left(1^{-}\right)$ arises not only in the processes with neutral current, which are considered above. The charged meson $M^{-}\left(0^{-}\right)=(d \bar{U})$ possesses the excited charged state too, $M^{-*}=M^{-}\left(1^{-}\right)$. The charged states $M^{-}$ and $M^{-*}$ arise in reaction with charged currents, for example, in the process of electron scattering on neutral meson $M^{0}=(u \bar{U})$ :

$$
e^{-} M^{0} \rightarrow v_{e} M^{-} ; v_{e} M^{-*}
$$

These reactions go through the exchange of $W$-boson in $t$-channel; that is, the lepton vertex in Equation (14) takes the form $\gamma_{\mu}\left(1-\gamma_{5}\right)$ and the hadron vertex $\Gamma_{\mu}$, in the general case, includes an additional form-factor $a_{-}\left(q^{2}\right)(e \cdot P)(P-K)_{\mu}$. Cross-section of the process $e^{-} M^{0} \rightarrow v_{e} M^{-}$was calculated in our previous work [21] for the case of low-energy scattering:

$$
\sigma\left(e^{-} M^{0} \rightarrow v_{e} M^{-}\right)=\frac{3 G_{F}^{2}\left|U_{u d}\right|^{2}}{8 \pi}\left(E_{e}+W\right)^{2}
$$

where $E_{e}$ is the energy of electron and $W=M v^{2} / 2$ is kinetic energy of non-relativistic $M$-particle.

To estimate the cross-section of electron elastic scattering on $M$-meson, it can be used the approximate analogy of this process with the scattering of electrons on the hydrogen-like atom. In the frame-work of HQET, heavy-light composite particle $M^{0}=(u \bar{U})$ is interpreted as heavy charged point-like particle (U-nuclear) at rest and light charged particle, $u$-quark, which is smeared around the center. For the case of electron scattering on the hydrogen atom, the expression of cross-section can be found in the Born approximation. At small scattering angles $(q a \ll 1)$, the expression for cross-section of the process $e M^{0} \rightarrow e M^{0}$ is $(e=\hbar=1)$ :

$$
d \sigma=q_{u}^{4} \alpha^{2} m_{e}^{2} a^{4} d \Omega
$$

where $q_{u}$ is the charge of up-type quark, $q_{u}=2 / 3$. Therefore, the value of cross-section strongly depends on the characteristic size of $M$-meson, $a$. From (18), it follows at $a \sim\left(10^{-13}-10^{-14}\right) \mathrm{cm}$ the value of differential cross-section $d \sigma / d \Omega \sim\left(1-10^{-4}\right) \mathrm{pb} / \mathrm{sr}$.

An important characteristics of the processes of exciting and recombination of $M$-mesons are fixed energy of state and the width of spectral line, which relate to the possibility of $\gamma$-rays registration. From preliminary analysis, it follows that hadronic DM at some conditions become not absolutely dark. To estimate the width of spectral line, we should calculate the matrix element of transition. As a result that the theory of such processes for the case of super-heavy hadrons is not developed, we again 
turn to the analogy with standard HL mesons $K, D, B$. In Ref. [14], one can find experimental data on the reactions corresponding to the process of recombination of excited states:

$$
K^{0 *}\left(1^{-}\right) \rightarrow K^{0}\left(0^{-}\right) \gamma ; D^{0 *}\left(1^{-}\right) \rightarrow D^{0}\left(0^{-}\right) \gamma ; B^{0 *}\left(1^{-}\right) \rightarrow B^{0}\left(0^{-}\right) \gamma
$$

The first reaction, where $K^{0 *}\left(1^{-}\right)=K^{*}(892)$, is the most informative: $B r\left(K^{*} \rightarrow K^{0} \gamma\right)=$ $2.46 \times 10^{-5}$ and $\Gamma^{\text {tot }}\left(K^{*}\right)=47.4 \mathrm{MeV}$, that is, $\Gamma\left(K^{*} \rightarrow K^{0} \gamma\right) \approx 0.7 \mathrm{keV}$. Taking into account heavy quark symmetry, which establishes an analogy in the mechanism of reactions for the cases of $K$ - and $M$-mesons, and using approximate relation of phase spaces, $K=\delta m /\left(m\left(K^{*}\right)-m\left(K^{0}\right)\right) \sim 10^{-5}$, we get rough estimation of the width of transition $\Gamma\left(M^{*} \rightarrow M^{0} \gamma\right) \sim 10^{-2} \mathrm{eV}$. From the data on the reaction $D^{0 *}\left(1^{-}\right) \rightarrow D^{0}\left(0^{-}\right) \gamma$, it follows the upper limit $\Gamma\left(M^{*} \rightarrow M^{0} \gamma\right)<10^{-2} \mathrm{keV}$, which does not contradict to the previous estimation. For the third reaction, $B^{0 *}\left(1^{-}\right) \rightarrow B^{0}\left(0^{-}\right) \gamma$, it is known only that this channel is dominant. Thus, above given brief analysis leads to the presence of narrow spectral lines, which can be registered as the manifestation of hyperfine splitting in HDM scenario.

Possible luminosity of dark (hidden) matter was intensively discussed in literature last year $[15,16,18]$ (and references therein). The most attention was given to the excess signals which were registered by underground experiment XENON1T [17]. Further, we briefly consider possibility of underground luminosity effect in the HDM scenario. First of all, we note that due to large cross-section of low-energy elastic $M N$-scattering, $\sigma \sim(0.1-1) \mathrm{b}$, free path of $M$-particles, $\lambda=1 /(\sigma n)$, is small. The value of $\lambda$ at the lower layer of atmosphere is an order of $10^{3} \mathrm{~m}$, and $0.1-1 \mathrm{~cm}$ in ground, which excludes the direct detection of DM particles by underground detectors. Initial kinetic energy of HDM particles $W_{k} \sim 10^{4} \mathrm{keV}$, where we have used the value of mass $m(M) \approx 10 \mathrm{TeV}$ and averaged velocity $v \approx 3 \times 10^{-3}$. As was shown in Ref. [21], the value of momentum transfer is very small in the process of collision of nucleon and $M$-particle due to strong inequality $m(N) \ll m(M)$. Therefore, $M$-particles reach the Earth's surface with nearly initial energy and enter to the process of fast underground thermalization. Due to repulsive character of $N M$-interaction potential the $M$-particles do not create coupled states with ordinary matter and drift to the center of Earth under gravitation. Dynamics of this process are not investigated, however, the presence of $M$-particles can manifest themselves as a result of excitation and recombination. As was shown above, energy scale of these processes is in the $\mathrm{keV}$ range, namely $E_{\gamma} \approx 2 \mathrm{kev}$ for the case of $M\left(0^{-}\right) \rightarrow M\left(1^{-}\right)$transitions. Here, we should note that this estimation depends on the value of mass, $m(M)$, and the accuracy of HQET predictions. Moreover, the mechanism of underground excitation is unknown, the most natural one is the interaction of neutrino with $M$-particles. To do more precise estimations, we should revise the cross-section of heavy quark annihilation at the freeze-out stage of evolution, directly calculate $1 / m_{Q}$ corrections to the spectrum of excited states in the frame-work of HQET and consider processes of exciting and recombination of new heavy mesons in more detail.

Now, we consider a possible manifestation of keV-signal, which is caused by hyperfine splitting, in the spectrum of X-rays from the galaxy clusters. In Refs. [27,28], it was reported about emission line at $E \approx 3.5 \mathrm{keV}$ in a spectrum of galaxy center and galaxy clusters. The nature and origin of 3.5-keV lines are the subject of considerable discussion so far (see, for instance, Refs. [29,30]). The simplest explanation of this line origin follows from the decay of dark matter candidates with mass ( $m \approx 7 \mathrm{keV})$ into two final photons, $\chi \rightarrow 2 \gamma$. In another scenario, the excited partner $\chi^{\prime}$ of DM particle $\chi$ is assumed mass-degenerated with the mass-splitting $\Delta m \sim 3 \mathrm{keV}$. The dark excited state then decays, $\chi^{\prime} \rightarrow \chi \gamma$ by the emission of monoenergetic photons with energy $E_{\gamma} \sim \Delta m$ [15]. Dark matter origin of this line is subjected to intensive discussion and criticism, see, for example, Ref. [30] where the evidence for the 3.5-keV feature in clusters was reconsidered. Here, we should note that the existence in nature of superheavy-light mesons inevitably (in the framework of HQET) leads to hyperfine mass-splitting of ground and excited levels. Transitions between these states generate emission of photons with energy $3.5 \mathrm{keV}$ when the mass of new heavy mesons $m(M) \approx 6 \mathrm{TeV}$. This estimation in the framework of HQET follows from Equation (8) without refering to DM hypothesis. This hypothesis together with relic density fixes the mass near $10 \mathrm{TeV}$, however, this value can be modified by account of the additional 
factors, for instance, coannihilation process. Moreover, as was noted in the introduction, the reference to DM is not obligatory, because new heavy quarks, such as singlet quark, hence heavy mesons, are predicted in the framework of the grand unification paradigm which generates an important class of SM extensions.

\section{Discussion and Conclusions}

Description of new particle interaction with usual matter and prediction of corresponding signals are the main purpose of the hidden matter study. Last time, luminosity of hidden sector and hyperfine splitting of spectrum are under considerable discussion. Here, we have considered the main theoretical and phenomenological consequences of the compositeness of new heavy hadrons. Composite particles usually have excited states with the spectrum which is defined by the nature and interactions of their components. The spectrum of excited states of new heavy hadrons was studied in full analogy with standard heavy-light mesons in the framework of HQET. We show that there are fine and hyperfine structures in the spectrum of states of new heavy hadrons. The existence of metastable charged heavy mesons is a consequence of fine mass-splitting $(\sim \mathrm{MeV})$ in the doublet of new heavy mesons $M=\left(M^{0}, M^{-}\right)$. Hyperfine mass-splitting $(\sim \mathrm{keV})$ of the ground and the lowest excited states follows from the heavy quark symmetry which is the basic idea of HQET. We have noticed, in this work, that effects of fine and hyperfine splitting do not depend on possible interpretation of new heavy hadrons as the DM candidates. This effect and its phenomenological manifestation always take place in the scenarios with heavy hadrons.

Principal phenomenological consequences of the fine and hyperfine splittings are briefly analyzed, in particular, manifestation of a metastable charged hadron and the luminosity of the hadronic hidden sector. The possibility of direct and indirect registration of heavy charged hadron in cosmic rays was considered. We show that the existence of the hyperfine structure leads to electromagnetic transitions and recombination. These processes are caused by the interactions of new hadrons with the ordinary matter and cosmic rays, and result in the effect of luminosity of the hadronic hidden sector. Note, the problem of luminous dark matter becomes actual in view of the results of XMM-Newton observations and underground experiment XENON1T. We have derived the restrictions on the parameters of new hadrons, which follow from the known cosmological constraints on the DM signals. These restrictions can be used in the forthcoming study of the particles physics beyond SM and their cosmological manifestation.

Author Contributions: The authors (V.K. and V.B.) contributed equally to the work. All authors have read and agreed to the published version of the manuscript.

Funding: Research was financially supported by Southern Federal University, 2020 (Ministry of Science and Higher Education of the Russian Federation) [In Gr /2020-03-IF].

Acknowledgments: Authors are sincerely grateful to M. Khlopov for fruitful cooperation in scientific research.

Conflicts of Interest: The authors declare no conflict of interest.

\section{References}

1. Huo, R.; Matsumoto, S.; Tsai, J.L.S.; Yanagida, T.T. A scenario of heavy but visible baryonic dark matter. JHEP 2016, 1609, 162-166. [CrossRef]

2. Luca, V.; Mitridate, A.; Redi, M.; Smirnov, J.; Strumia, A. Colored Dark Matter. Phys. Rev. D 2018, 97, 115024. [CrossRef]

3. Beylin, V.; Kuksa, V. Dark Matter in the Standard Model Extention with Singlet Quark. Adv. High Energy Phys. 2018, 2018, 8670954. [CrossRef]

4. Beylin, V.; Kuksa, V. Possibility of hadronic dark matter. Int. J. Mod. Phys. D 2018, 28, 1941001. [CrossRef]

5. Aprile, E.; Aalbers, J.; Agostini, F.; Alfonsi, M.; Amaro, F.D.; Anthony, M.; Arneodo, F.; Barrow, P.; Baudis, L.; Bauermeister, B.; et al. First Dark Matter Search Results from the XENON1 Experiment. Phys. Rev. Lett. 2017, 119, 181301. [CrossRef] [PubMed] 
6. Beylin, V.; Khlopov, M.Y.; Kuksa, V.; Volchanskiy, N. Hadronic and Hadron-Like Physics of Dark Matter. Symmetry 2019, 11, 587. [CrossRef]

7. Bazhutov, Y.N.; Vereshkov, G.M.; Kuksa, V.I. Experimental and Theoretical Premises of New Stable Hadron Existence. Int. J. Mod. Phys. A 2017, 2, 1759188. [CrossRef]

8. Haslbauer, M.; Banik, I.; Kroupa, P. The KBC void and Hubble tension contradict $\Lambda$ CDM on a Gpc scale-Milgromian dynamics as a possible solution. Mon. Not. R. Astron. Soc. 2020, 499, 2845-2883. [CrossRef]

9. Isgur, N.; Wise, M.B. Spectroscopy with heavy-quark symmetry. Phys. Rev. Lett. 1991, 66, 1130-1133. [CrossRef]

10. Flynn, J.N.; Isgur, N. Heavy quark symmetry: Ideas and applications. J. Phys. G 1992, 18, 1627-1644. [CrossRef]

11. Neubert, M. Heavy-quark symmetry. Phys. Rep. 1994, 245, 259-395. [CrossRef]

12. Mannel, T. Effective Theory for Heavy Quarks. Rep. Prog. Phys. 1997, 60, 1113. [CrossRef]

13. Ebert, D.; Galkin, V.O.; Faustov, R.N. Mass spectrum of orbitally and radially excited heavy-light mesons in the relativistic quark model. Phys. Rev. D 1998, 57, 5663-5669. [CrossRef]

14. Tanabashi, M.; Hagiwara, K.; Hikasa, K.; Nakamura, K.; Sumino, Y.; Takahashi, F.; Tanaka, J.; Agashe, K.; Aielli, G.; Amsler, C.; et al. The Review of Particle Physics (2018). Phys. Rev. D 2018, 98, 1. [CrossRef]

15. Bell, N.F.; Dent, J.B.; Dutta, B.; Ghosh, S.; Kumar, J.; Newstead, J.L. Explaining the XENON1T excess with Luminous Dark Matter. arXiv 2020, arXiv:2006.12461.

16. Lee, H.M. Exotermic Dark Matter for XENON1T Excess. arXiv 2020, arXiv:2006.13183.

17. Aprile, E.; Aalbers, J.; Agostini, F.; Alfonsi, M.; Althueser, L.; Amaro, F.D.; Antochi, V.C.; Angelino, E.; Angevaare, J.R.; Arneodo, F.; et al. Observation of Excess Electronic Recoil Events in XENON1T. arXiv 2020, arXiv:2006.09721.

18. Baryakhtar, M.; Berlin, A.; Liu, H.; Weiner, N. Electromagnetic Signals of Inelastic Dark Matter Scattering. arXiv 2020, arXiv:2006.13918.

19. Poulin, V.; Lesgourgues, J.; Serpico, P.D. Cosmological constraints on exotic injection of electromagnetic energy. JCAP 2017, 3, 043. [CrossRef]

20. Acharya, S.K.; Khatri, R. CMB anisotropy and BBN constrains on pre-recombination decay of dark matter to visible particles. JCAP 2019, 12, 046. [CrossRef]

21. Kuksa, V.I.; Beylin, V.A. Hyperfine Splitting of Excited States of New Heavy Hadrons and Low-energy Interaction of Hadronic Dark Matter with Photons, Nucleons, and Leptons. Universe 2020, 6, 84. [CrossRef]

22. Cyburt, R.H.; Fields, B.D.; Pavlidou, V.; Wandelt, B.D. Constraining Strong Baryon-Dark Matter Interactions with primordial Nucleosynthesis and Cosmic Rays. Phys. Rev. D 2002, 65, 123503. [CrossRef]

23. Erickcek, A.L.; Steinhardt, P.J.; McCammon, D.; McGuire, P.C. Constraints on the interactions between Dark Matter and Baryons from the X-ray Quantum Calorimetry Experiment. Phys. Rev. D 2007, 76, 042007. [CrossRef]

24. Mack, G.D.; Manohar, A. Closing the window on high-mass stongly interacting dark matter. J. Phys. 2013, 40, 11. [CrossRef]

25. Baryakhtar, M.; Bramante, J.; Li, S.W.; Linden, T.; Raj, N. Dark Kinetic Heating of Neutron Stars and An Infrared Window on WIMPs, SIMPs, and Pure Higgsinos. Phys. Rev. Lett. 2017, 119, 131801. [CrossRef]

26. Baric, N.; Dash, P.C. Exclusive semileptonic decay of $D$ and $B$ mesons in the independent quark model. Phys. Rev. D 1996, 53, 1366-1377. [CrossRef]

27. Bulbul, E.; Markevitch, M.; Foster, A.; Smith, R.K.; Loewenstein, M.; Randall, S.W. Detection of an unidentified emission line in the stacked X-ray spectrum of Galaxy clusters. Astrophys. J. 2014, $789,27$. [CrossRef]

28. Boyarsky, A.; Ruchayskiy, O.; Iakubovskyi, D.; Franse, I. An unidentified line in X-ray spectra of the Andromeda galaxy and Perseus galaxy cluster. Phys. Rev. Lett. 2014, 113, 251301. [CrossRef] 
29. Boyarsky, A.; Drewes, M.; Lasserre, T.; Merteus, T. The origin of $3.5 \mathrm{keV}$ emission line remains debated. Prog. Part. Nucl. Phys. 2019, 1, 104.

30. Bhargava, S.; Giles, P.A.;Romer, A.K.; Jeltema, T.; Mayers, J.; Bermeo, A.; Hilton, M.; Wilkinson, R.; Vergara, C.; et al. The XMM Cluster Survey: New evidence for the $3.5-\mathrm{keV}$ feature in clusters is inconsistent with a dark matter origin. MNRAS 2020, 497, 656-671. [CrossRef]

Publisher's Note: MDPI stays neutral with regard to jurisdictional claims in published maps and institutional affiliations.

(C) 2020 by the authors. Licensee MDPI, Basel, Switzerland. This article is an open access article distributed under the terms and conditions of the Creative Commons Attribution (CC BY) license (http:/ / creativecommons.org/licenses/by/4.0/). 